\title{
Novel FGFR2-INA fusion identified in two low-grade mixed neuronal-glial tumors drives oncogenesis via MAPK and PI3K/mTOR pathway activation
}

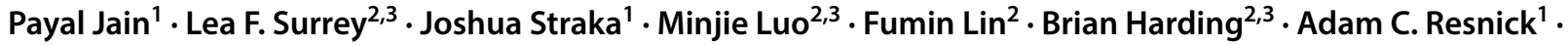 \\ Phillip B. Storm ${ }^{4}$. Anna Maria Buccoliero ${ }^{5}$ Mariarita Santi ${ }^{2,3} \cdot$ Marilyn M. Li $^{1,2,3,6}$. Angela J. Waanders ${ }^{1,6,7}$
}

Received: 9 February 2018 / Revised: 8 May 2018 / Accepted: 8 May 2018 / Published online: 16 May 2018

(c) The Author(s) 2018

As a group, mixed neuronal-glial tumors (MNGTs) exhibit genetic variability, including stable genomes, whole chromosome gains, $B R A F-\mathrm{V} 600 \mathrm{E}$, and $F G F R 1$ mutations $[8,9,11$, 12]. While histologic criteria are described to distinguish MNGT types ganglioglioma (GG) and dysembryoplastic neuroepithelial tumor (DNT), non-specific features preclude confident classification in a high proportion of cases $[2,8$, $10,12]$. Herein, we report the characterization of a novel FGFR2-INA fusion gene identified during clinical genomic profiling in two cases of MNGTs that could not be specifically classified as GG or DNT.

Clinical, imaging, histology, and fusion gene characteristics of each case are summarized in suppl. Table 1 (Online Resource 1). Both patients presented with seizures, cortical-based tumors, and one patient's tumor was recurrent. By histology and immunohistochemistry, both cases

Payal Jain and Lea F. Surrey are co-first authors and contributed equally.

Marilyn M. Li and Angela J. Waanders are co-last authors and contributed equally.

Electronic supplementary material The online version of this article (https://doi.org/10.1007/s00401-018-1864-5) contains supplementary material, which is available to authorized users.

Angela J. Waanders

WAANDERSA@email.chop.edu

1 Center for Data Driven Discovery in Biomedicine, Children's Hospital of Philadelphia, 3501 Civic Center Boulevard, Philadelphia, PA 19104, USA

2 Department of Pathology and Laboratory Medicine, Children's Hospital of Philadelphia, Philadelphia, PA 19104, USA

3 Department of Pathology and Laboratory Medicine, Perelman School of Medicine, University of Pennsylvania, Philadelphia, PA, USA consisted of oligodendrocyte-like cells and admixed neurons within microcytic spaces (Fig. 1a). GFAP-positive astrocytes, CD34 expression (MNGT-1), and calcification were observed. Both cases lacked pools of mucin, floating neurons, specific glioneuronal elements, eosinophilic granular bodies, and perivascular inflammation. Features were most similar to DNT; however, both lacked key criteria for this diagnosis.

Targeted RNA-sequencing revealed a novel in-frame fusion between FGFR 2 exon 17 and INA exon 2 (Fig. 1b) in both cases. Additional DNA sequence and copy number variants of clinical significance were also identified by targeted next-generation sequence panel [suppl. Tables 2, 3, 4 (Online Resource 1)] [7]. FGFR2, a receptor kinase, regulates several growth-related signaling pathways implicated in cancer progression, including RAS-RAF-MAPK and $\mathrm{PI} 3 \mathrm{~K} / \mathrm{AKT} / \mathrm{mTOR}$ [3]. INA encodes the alpha-internexin protein involved in cytoskeletal organization and neuronal morphogenesis [6]. The novel fusion retains the extracellular immunoglobin-like and tyrosine kinase domains of FGFR2, suggesting oncogenic activation of downstream signaling, and the truncated coil 2 and tail region of INA, suggesting dimerization (Fig. 1c).

4 Department of Neurosurgery, Children's Hospital of Philadelphia, Philadelphia, PA 19104, USA

5 Meyer Children's Hospital, Florence, Italy

6 Department of Pediatrics, Perelman School of Medicine University of Pennsylvania, Philadelphia, PA 19104, USA

7 Division of Oncology, Children's Hospital of Philadelphia, Philadelphia, PA 19104, USA 
(a)

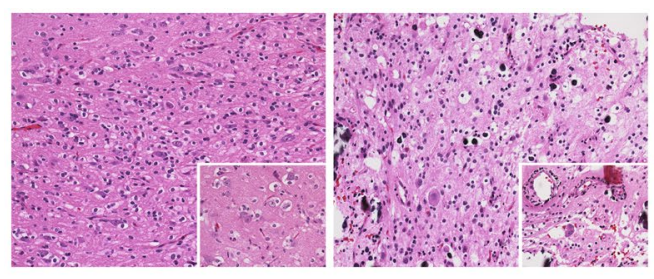

(b)

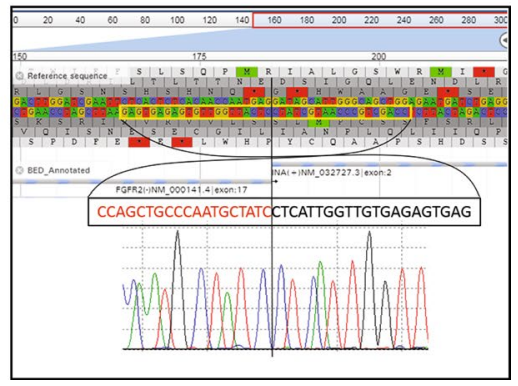

(d)

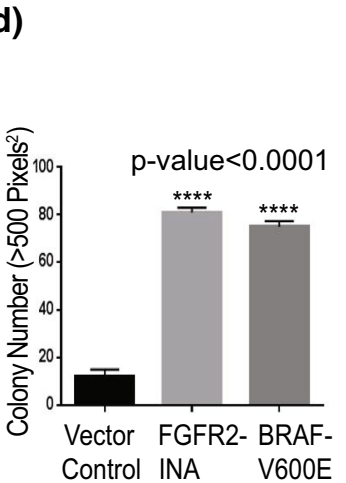

(e) (f)

(c)

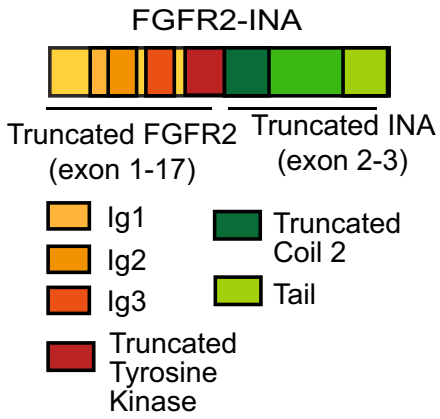

(g)

Everolimus $\mu \mathrm{M} \quad 0 \quad 10 \quad 10 \quad 10$

Trametinib $\mu \mathrm{M} \quad 00.0050 .010 .1$

PMEK

pERK

pAKT ${ }^{\mathrm{S}} 73$

pS6

tMEK

tERK

tAKT

tS6

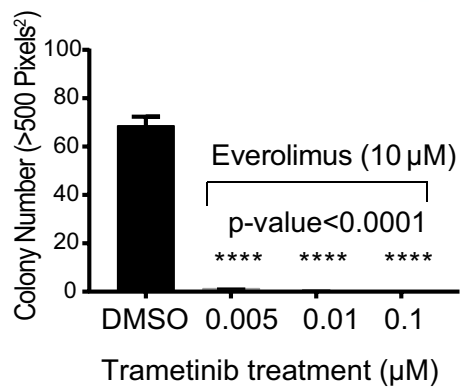

Fig. 1 Histologic and sequencing characteristics of two MNGT harboring an FGFR2-INA fusion that activates the MAPK and PI3 K/ mTOR pathways. a MNGT-1 (left) and MNGT-2 (right) contained small oligodendrocyte-like cells admixed with neurons surrounded by clear microcystic spaces (insets, 400X H\&E), 200X H\&E. b RNAseq reads and confirmatory reverse complement Sanger sequencing of FGFR2-INA. c Structure of FGFR2-INA: FGFR2 exons 2-3 encode Ig-1, exons 4-5 encode Ig-2, exons 6-7 encode Ig-3 domains, and exons 9-17 encode a truncated tyrosine kinase domain (lacking three

We cloned FGFR2-INA and stably expressed it in NIH/3T3 and Tp53-null primary mouse astrocytes (PMAs) $[1,5]$ [suppl. Figure 1 (Online Resource 2)]. In soft agar proliferation assays, FGFR2-INA expressing NIH/3T3 showed a significant increase in colony count over control, similar to BRAF V600E $(p<0.0005)$ (Fig. 1d). Next, we assessed the signaling potential of FGFR2-INA. In serum starved conditions, we observed high-level activation of both the MAPK and PI3 K/mTOR pathways assessed via elevated levels of phosphorylated-ERK and -S6, respectively, compared to vector-controlled cells (Fig. 1e). Mechanistically, we found that FGFR2-INA homo-dimerizes in amino acids from FGFR2 exon-18). d Soft agar assay using NIH3T3 stably expressing FGFR2-INA, $n=10$. Error bars represent SEM. e Western blot analysis of MAPK and PI $3 \mathrm{~K} / \mathrm{mTOR}$ pathway proteins in NIH3T3 and PMAs. 'p'-phosphorylated; ' $t$ '-total protein. $\mathbf{f}$ Co-immunoprecipitation (Co-IP) assay with anti-Myc tag beads and co-transfecting HEK293 cells with Flag (F)- and Myc (M)-tagged FGFR2-INA, and F-FGFR2-INA with M- vector control. g Effect of combinatorial trametinib and everolimus treatment on FGFR2-INAdriven oncogenic signaling and growth in NIH3T3 cells

co-immunoprecipitation assays suggesting dimerizationinduced activation of FGFR2-INA (Fig. 1f). Using combinatorial targeting of downstream MAPK and PI3K/mTOR pathways with trametinib and everolimus, respectively, we could suppress FGFR2-INA-driven oncogenic signaling and growth (Fig. 1f, suppl. Figure 2 (Online Resource 3)).

We identify and characterize a novel FGFR2-INA fusion associated with unclassified MNGT in two patients lacking other reported driver alterations (BRAF-V600E and $F G F R 1$ ). Other $F G F R 2$ fusions have been identified in epileptogenic tumors of the young with some overlapping histologic features to the current two cases [4]. It is possible that these 
tumors represent an emerging category of low-grade epileptogenic tumor. Our functional studies show that the FGFR2INA fusion drives oncogenesis potentially via activation of the MAPK and PI3 K/mTOR pathways. Therefore, FGFR2INA is the likely driver of tumorigenesis in at least a subset of MNGTs and is a potential target for small-molecule inhibitors.

Acknowledgements This work was supported by Children's Brain Tumor Tissue Consortium (CBTTC).

Open Access This article is distributed under the terms of the Creative Commons Attribution 4.0 International License (http://creativeco mmons.org/licenses/by/4.0/), which permits unrestricted use, distribution, and reproduction in any medium, provided you give appropriate credit to the original author(s) and the source, provide a link to the Creative Commons license, and indicate if changes were made.

\section{References}

1. Bandopadhayay P, Ramkissoon LA et al (2016) MYB-QKI rearrangements in angiocentric glioma drive tumorigenicity through a tripartite mechanism. Nat Genet 48:273-282. https://doi. org/10.1038/ng. 3500

2. Hirose T, Scheithauer BW (1998) Mixed dysembryoplastic neuroepithelial tumor and ganglioglioma. Acta Neuropathol 95:649-654

3. Holbrook JD, Parker JS et al (2011) Deep sequencing of gastric carcinoma reveals somatic mutations relevant to personalized medicine. J Transl Med 9:119. https://doi. org/10.1186/1479-5876-9-119

4. Huse JT, Snuderl M et al (2017) Polymorphous low-grade neuroepithelial tumor of the young (PLNTY): an epileptogenic neoplasm with oligodendroglioma-like components, aberrant CD34 expression, and genetic alterations involving the MAP kinase pathway. Acta Neuropathol 133:417-429. https://doi.org/10.1007/ s00401-016-1639-9

5. Jain P, Fierst TM et al (2017) CRAF gene fusions in pediatric lowgrade gliomas define a distinct drug response based on dimerization profiles. Oncogene 36:6348-6358. https://doi.org/10.1038/ onc. 2017.276

6. Kaplan MP, Chin SS, Fliegner KH, Liem RK (1990) Alphainternexin, a novel neuronal intermediate filament protein, precedes the low molecular weight neurofilament protein (NF-L) in the developing rat brain. J Neurosci 10:2735-2748

7. Li MM, Datto M et al (2017) Standards and guidelines for the interpretation and reporting of sequence variants in cancer: a joint consensus recommendation of the association for molecular pathology, American Society of Clinical Oncology, and College of American Pathologists. J Mol Diagn 19:4-23. https://doi. org/10.1016/j.jmoldx.2016.10.002

8. Louis DN, Perry A et al (2016) The 2016 World Health Organization Classification of Tumors of the Central Nervous System: a summary. Acta Neuropathol 131:803-820. https://doi. org/10.1007/s00401-016-1545-1

9. Prabowo AS, van Thuijl HF et al (2015) Landscape of chromosomal copy number aberrations in gangliogliomas and dysembryoplastic neuroepithelial tumours. Neuropathol Appl Neurobiol 41:743-755. https://doi.org/10.1111/nan.12235

10. Prayson RA (1999) Composite ganglioglioma and dysembryoplastic neuroepithelial tumor. Arch Pathol Lab Med 123:247-250 (10.1043/0003-9985(1999)123<0247:cgadnt>2.0.co;2).

11. Qaddoumi I, Orisme W et al (2016) Genetic alterations in uncommon low-grade neuroepithelial tumors: BRAF, FGFR1, and MYB mutations occur at high frequency and align with morphology. Acta Neuropathol 131:833-845. https://doi.org/10.1007/s0040 1-016-1539-Z

12. Stone TJ, Keeley A et al (2018) Comprehensive molecular characterisation of epilepsy-associated glioneuronal tumours. Acta Neuropathol 135:115-129. https://doi.org/10.1007/s0040 1-017-1773-z 